SHEP-09-29

DFTT 70/2009

October 31, 2018

\title{
Strong and Electro-Weak Supersymmetric Corrections to Single Top Processes at the Large Hadron Collider
}

\author{
G. Macorini ${ }^{1}$, S. Moretti ${ }^{2,3}$ and L. Panizzi ${ }^{4}$ \\ ${ }^{1}$ Dipartimento di Fisica, Università del Salento and INFN, \\ Via Arnesano, 73100 Lecce, Italy. \\ ${ }^{2}$ School of Physics and Astronomy, University of Southampton, \\ Highfield, Southampton SO17 1BJ, UK. \\ ${ }^{3}$ Dipartimento di Fisica Teorica, Università di Torino, \\ Via Pietro Giuria 1, 10125 Torino, Italy. \\ ${ }^{4}$ Université de Lyon, France; Université Lyon 1, CNRS/IN2P3, \\ UMR5822 IPNL, F-69622 Villeurbanne Cedex, France.
}

\begin{abstract}
We present the one-loop corrections originating from Quantum Chromo-Dynamics (QCD) and Electro-Weak (EW) interactions of Supersymmetric (SUSY) origin within the Minimal Supersymmetric Standard Model (MSSM) to the single-top processes $b q \rightarrow t q^{\prime}$ and $q \bar{q}^{\prime} \rightarrow t \bar{b}$. We illustrate their impact onto top quark observables accessible at the Large Hadron Collider (LHC) in the ' $t+$ jet' final state, such as total cross section, several differential distributions and left-right plus forward-backward asymmetries. We find that in many instances these effects can be observable for planned LHC energies and luminosities, quite large as well as rather sensitive to several MSSM parameters.
\end{abstract}




\section{Introduction}

Top quark processes at the LHC will be an ideal laboratory where it will be possible to profile with great accuracy the heaviest particle of the Standard Model (SM) and eventually extract possible effects of New Physics (NP) Beyond the SM (BSM). The manifestation of NP at the LHC will in fact depend on the actual NP mass scale, $M_{\mathrm{NP}}$. If the available energy is less than $M_{\mathrm{NP}}$, then NP will manifests itself through virtual effects. Conversely, if the available energy is greater than $M_{\mathrm{NP}}$, NP will appear through the production and decay of new real particle resonances. A mixture of both scenarios may of course also occur.

If NP is identified with SUSY, then it is fair to say, based on the available literature, that much has been done in the second respect. Various methods to extract the presence of new resonant SUSY particles have in fact been devised over the years and applied to several possible model realisations of a SUSY theory, particularly for the case of the simplest SUSY scenario, the MSSM. In constrast, much less has been done so far in the first respect. Primarily because the LHC is perceived not to be a precision machine, due to both the undefined partonic energy and the intrinsically large hadronic background, so that virtual effects (of order $\alpha_{s}$ and $\alpha$ or indeed smaller) are expected to be not easily discernible in the data. However, there are certain processes where the experimental precision is expected to eventually become comparable to the size of the virtual effects of NP.

Among these processes, one can certainly list top quark production, both in doubleand single-top mode. In fact, with typical hadro-production cross sections at the LHC of order $800 \mathrm{pb}$ and $300 \mathrm{pb}$ respectively (at $14 \mathrm{TeV}$ ) and collider luminosities that can reach the $300 \mathrm{fb}^{-1}$ level, several hundred million top events will altogether be produced during the lifetime of the CERN machine. Clearly, on the one hand, this renders the statistical error applicable on the experimental side to typical top quark observables negligible. Furthermore, on the other hand, the main systematics affecting the latter (both theoretical, coming from the Parton Distribution Function (PDF) dependence, and experimental, coming from the jet energy scale uncertainty) will be understood at the percent level after the first hadronic data samples will have been collected and studied in detail.

Therefore, both double- and single-top production processes at the LHC lend themselves to being precision physics laboratories, where, in particular, virtual effects of SUSY (when $M_{\mathrm{NP}} \equiv M_{\mathrm{SUSY}} \gg m_{t}$, where $m_{t}$ is the top quark mass) can possibly be extracted, in the ultimate attempt to understand the underlying dynamics of SUSY breaking. We intend to illustrate here the potential afforded in this respect by two single-top production processes, namely the t-channel:

$$
b q \rightarrow t q^{\prime}
$$

and the s-channel:

$$
q \bar{q}^{\prime} \rightarrow t \bar{b}
$$


(plus, of course, their charge conjugated channels), where $q^{\left({ }^{\prime}\right)}$ represents a light quark $(u, d, s$ or $c)$

The plan of the paper is as follows. The next section describes what is available in current literature concerning single-top processes at the LHC in terms of their higher order corrections within the MSSM. Sect. 3 illustrates how we performed the calculation of the one-loop SUSY QCD and EW corrections to the two single-top channels (1)-(2). The following section presents our numerical results. We conclude in Sect. 5 ,

\section{Single-top Processes at the LHC}

Due to the their relevance for LHC physics, single- as well as double-top processes have extensively been studied in previous years, with a twofold aim: on the one hand, in order to have a precise SM prediction, at least at the complete NLO (and possibly next-to-NLO (NNLO) for the QCD corrections); on the other hand, in order to investigate possible deviations from SM predictions due to the presence of NP. These two goals are obviously related, the former being a precondition for the second: in order to extract meaningful information from NP processes entering at one-loop level top quark hadro-production at the LHC, clearly, all similar SM effects should be well under control.

One-loop SM corrections to single- and double-top production in hadron-hadron collisions have been known for some time. These include the SM QCD [1] and SM EW [2] ones to $g g, q \bar{q} \rightarrow t \bar{t}$ (double-top production) and the corresponding ones [3, 4] for $b q \rightarrow t q^{\prime}$, $q \bar{q}^{\prime} \rightarrow t \bar{b}$ and $b g \rightarrow t W^{-}$(single-top production). Both SM QCD and EW corrections can be large, although in complementary energy regimes: $\sqrt{s} \approx m_{t}$ and $\sqrt{s} \gg m_{t}$, respectively. However, they all are rather stable against variations of the factorisation and/or renormalisation scales (these in turn quantifying the systematic uncertanties related to the unknown two-loop, or even higher order, corrections), so that one can conceivably attempt to investigate virtual effects of some NP, particularly of SUSY origin, induced onto single- and double-top processes. To stay with the MSSM, QCD and EW corrections to double-top production have been quantified in [5] and [6], respectively, whilst only part of these are known to date for the case of single-top reactions [7.

Our paper is thus the first one where both MSSM QCD and EW corrections to singletop processes inducing a ' $t$ plus jet' signature in the final state are computed (i.e., $b q \rightarrow t q^{\prime}$

and $q \bar{q}^{\prime} \rightarrow t \bar{b}$ ). It is in fact from this perspective that we decided to postpone to another publication the presentation of similar corrections to the third single-top channel (i.e., $\left.b g \rightarrow t W^{-}\right)$, in the sense that the latter originates an altogether different signature in the final state, 't plus lepton and missing (transverse) energy' or ' $t$ plus two jets', depending on whether the $W^{ \pm}$boson produced in association with the top quark decays leptonically or hadronically. In fact, both of the latter require different triggers and selection procedures with respect to the former, so that, from a phenomenological point of view, they deserve 
a separate treatment.

\section{Calculation}

The values of the SM input parameters considered for the numerical evaluation of the one-loop corrections can be found in Tab. 1.

\begin{tabular}{|c|cc|}
\hline Coupling constants & $\alpha\left(M_{Z}\right)=1 / 127.93400652$ & $\alpha_{s}\left(M_{Z}\right)=0.1176$ \\
\hline Gauge boson masses & $M_{W}=80.424 \mathrm{GeV}$ & $M_{Z}=91.1876 \mathrm{GeV}$ \\
\hline Heavy Quark Masses & $m_{t}=170.9 \mathrm{GeV}$ & $m_{b}^{\overline{M S}}\left(m_{b}\right)=4.2 \mathrm{GeV}$ \\
\hline
\end{tabular}

Table 1: Numerical values of SM inputs.

We used the CTEQ6 2006 PDF set [8], in particular the CTEQ6L fit, which is LO in QCD, choosing renormalisation and factorisation scales both equal to the top mass, $m_{t}$.

As intimated in the introduction, due to the relatively large single-top production cross sections (in particular for the t-channel process) and the consequent large available statistics, we reckon that it will be possible at the LHC to perform "precision physics" of top samples produced singly. It is therefore a worthy task trying to understand systematically if sizable virtual effects could modify the SM predictions, providing an indirect but encouraging hint of NP. As a first step we performed an adaptive scan over both the MSSM and minimal-SUper GRAvity (mSUGRA) parameter spaces in order to investigate whether there exist regions with large one-loop corrections. We have done so limitedly to the inclusive production cross sections in either channel.

While in the MSSM case we have input the relevant parameters directly at the EW scale, in the mSUGRA scenario the low energy spectra have been obtained evolving the input parameters from the Grand Unification Theory (GUT) scale down to the EW scale through the code SUSPECT [9].

We have developed a dedicated $\mathrm{C}++$ code to compute the one-loop corrections and, as an internal check, we have tested the cancellation of Ultra-Violet (UV) divergences appearing in the loop integrations. To quantify the one-loop corrections, we will mainly focus on $K$-factors, defined in general as the ratio NLO/LO of a given observable, with all relevant quantities (QCD and EW coupling constants, etc.) evaluated at the given order consistently. The PDFs, on the other hand, have been kept at LO since there NLO effects are of SM origin, whereas we are looking here at purely SUSY corrections, therefore using NLO QCD PDF sets would introduce spurious SM effects from higher orders.

Guided by the inclusive results, we have then looked at differential distributions for some 'benchmark points'. In particular, alongside the typical kinematical observables (invariant masses, transverse momenta, (pseudo)rapidities), due to the possibility of measuring spin-related observables in top samples (the top (anti)quark decays before hadro- 
nising in fact, thus efficiently transmitting its polarisation state onto the ensuing decay products), we have also focused our attention on the following observables:

- the Left-Right Asymmetry $\left(A_{\mathrm{LR}}\right)$;

- the Forward-Backward Asymmetry $\left(A_{\mathrm{FB}}\right)$, only for the s-channel.

$A_{\mathrm{LR}}$ is defined as usual by the following ratio:

$$
A_{\mathrm{LR}}=\frac{\sigma_{p p \rightarrow t_{L}+X}-\sigma_{p p \rightarrow t_{R}+X}}{\sigma_{p p \rightarrow t_{L}+X}+\sigma_{p p \rightarrow t_{R}+X}}
$$

$A_{\mathrm{FB}}$ deserves further details, since the definition of "Forward" and "Backward" regions in a $p p$ collider is not straightforward. In the s-channel process the initial state can essentially be $u \bar{d}$ or $c \bar{s}$. In the first case $u$ is a valence quark, and thus the momentum fraction it carries is much bigger than that of the $\bar{d}$ sea-quark. It is then possible to reconstruct here the direction of the incoming valence quark just by identifying the direction of the boosted top in the final state. This is a statistical process, since s-channel single-top production can be initiated by two sea-quarks too, such as in the $c \bar{s}$ case, and the weight of the various events is given by the relevant PDF (hereafter denoted as $P$ ). The definition of $A_{\mathrm{FB}}$ is then given by the following relation [10]:

$$
\begin{aligned}
A_{\mathrm{FB}}= & \frac{1}{\int d x_{1} d x_{2} \sum_{q=u, d, c, s}\left(P_{q}\left(x_{1}, \mu\right) P_{\bar{q}}\left(x_{2}, \mu\right)+P_{\bar{q}}\left(x_{1}, \mu\right) P_{q}\left(x_{2}, \mu\right)\right.} \times \\
& \int\left[d x_{1} d x_{2} \hat{\sigma}(p p \stackrel{u \bar{d}}{\rightarrow} t \bar{b}+X)\right. \\
& \left.\left(P_{u}\left(x_{1}, \mu\right) P_{\bar{d}}\left(x_{2}, \mu\right)-P_{\bar{d}}\left(x_{1}, \mu\right) P_{u}\left(x_{2}, \mu\right)\right) \operatorname{sign}\left(x_{1}-x_{2}\right)\right]
\end{aligned}
$$

where $\mu$ is the factorisation/renormalisation scale.

We also have computed differential distributions of these quantities over the invariant mass of the final state, the transverse momentum of the top as well as the rapidity of both the top and the light quark in the final state.

Coming back to the scans, in the general MSSM scenario, we have explored over all soft parameters involved in the generation of the spectra, while in the mSUGRA scenario we have scanned over the four standard mSUGRA input parameters. The scans have been performed interfacing the tool adScan[1] to our code.

The main result of this preliminary analysis, both for MSSM and mSUGRA, is that the relative effect of the SUSY one-loop corrections seems to be rather small over the whole range of the independent parameters. Moreover, the effects show a very smooth and mild dependence on the parameters. A meaningful sample of our results is shown in Fig. 1. Each point in the diagrams corresponds to a full computation of NLO effects for a point in the parameter space, plotted versus a low energy parameter, such as a 
mass or mixing angle evaluated at the EW scale. We have chosen to show just three of them, corresponding to the parameters which are expected to affect most heavily the NLO corrections: $m_{\tilde{g}}$ (the gluino mass), $\tan \beta$ (the ratio of the two doublet Higgs vacuum expectation values) and $m_{\tilde{t}_{1}}$ (the lightest stop mass).

From the $\tan \beta$ plot it is easy to see that the density of the points is larger for very small values of the corrections and rapidly decreases for larger values of the effect. Furthermore, and possibly contrary to naive expectations, the density of the points is almost $\tan \beta$ independent. In essence, for the largest part of the parameters space, the corrections are very small, below the $2 \%$ level, while it is unlikely to find parameters configurations leading to corrections as high as $5 \%$, and this result holds independently of $\tan \beta$ (i.e., there are no visible regions in the $\tan \beta$ range where one-loop effects have a maximum). Trivially, the plots of the corrections as a function of the gluino or stop masses simply state that it is more likely to find "larger" (6\% or so at the most) corrections in parameters regions where these masses are small.

Despite the results obtained from the scan are never very large, it should be noted that these refer to inclusive corrections only. However, while scanning over the MSSM and mSUGRA parameter spaces, we have also tested for the size of SUSY QCD and EW corrections to differential distributions (including asymmetries) and found them to be rather large, albeit in limited (and at times disfavoured) regions of phase space, thereby explaining their smallness in the inclusive results. In order to illustrate the typical pattern emerging at differential level, we have defined a couple of representative benchmark mSUGRA points, whose parameters are given in Tab. 2 .

\begin{tabular}{|c|ccccc|}
\hline mSUGRA scenario & $m_{0}$ & $m_{1 / 2}$ & $A_{0}$ & $\tan \beta$ & $\operatorname{sign} \mu$ \\
\hline LS2 & 300 & 150 & -500 & 50 & + \\
SPS1a & 100 & 250 & -100 & 10 & + \\
\hline
\end{tabular}

Table 2: Input parameters for the mSUGRA benchmark points (all values with mass dimension are in $\mathrm{GeV}$ ).

The SPS1a point 12 is the typical mSUGRA "standard candle" while the LS2 point has been introduced in [13] and it is characterised by an interestingly light spectrum (yet compliant with current experimental and theoretical constraints), which - if realised in Nature - could prompt for easily detectable signals at the LHC. More details about the two spectra can be found in Fig. 2 .

\section{Results}

It has recently been scheduled a long LHC run at $7 \mathrm{TeV}$, which will probably last two years and will hopefully lead directly to the final expected energy of $14 \mathrm{TeV}$. In view 
of this recent development, we present our results for both such planned energies. Our numerical results will be illustrated in turn in the following two sub-sections. Notice that we will not show all the differential distributions analysed for the two benchmark points, rather we will illustrate a meaningful sample of these (and only in the $14 \mathrm{TeV}$ case) and focus on the one-loop corrections they receive. We will also show the contribution of the SUSY EW and QCD parts separately, in some instances at least, to better understand the features of the underlying dynamics: like, e.g., dominant contributions, asymptotic behaviours and threshold effects.

\subsection{LHC at $7 \mathrm{TeV}$}

The total cross section and asymmetries for t- and s-channel are given in Tabs. 3 and 4 respectively.

\begin{tabular}{|c|c|c|}
\hline & $\sigma(\mathrm{pb})$ & L-R Asymmetry $(1-\delta)$ \\
\hline Born & 35.44 & $\delta=4.7327 \times 10^{-7}$ \\
\hline LS2 & $35.29(K=0.996)$ & $\delta=4.7515 \times 10^{-7}\left(1-K=1.87 \times 10^{-9}\right)$ \\
SPS1 & $35.33(K=0.997)$ & $\delta=4.7353 \times 10^{-7}\left(1-K=2.6 \times 10^{-10}\right)$ \\
\hline
\end{tabular}

Table 3: Numerical results for t-channel production at $7 \mathrm{TeV}$.

\begin{tabular}{|c|c|c|c|}
\hline & $\sigma(\mathrm{pb})$ & L-R Asymmetry & F-B Asymmetry \\
\hline Born & 2.061 & 0.6777 & 0.598049 \\
\hline LS2 & $2.086(K=1.0121)$ & $0.6796(K=1.0028)$ & $0.598018(K=0.999948)$ \\
SPS1 & $2.062(K=1.0005)$ & $0.6783(K=1.0009)$ & $0.598046(K=0.999995)$ \\
\hline
\end{tabular}

Table 4: Numerical results for s-channel production at $7 \mathrm{TeV}$.

As it is possible to see, in both the two channels, the NLO corrections to the total cross sections are opposite in sign: in the t-channel case one-loop corrections reduce the cross section, while in the s-channel there is an enhancement, but the corrections are $1 \%$ or less in both the benchmarks considered (in line with the results from the mSUGRA scans). The main difference between the two channels is of course the value of the cross section, $\sigma$. Focusing on t-channel, which has a greater $\sigma$, we have found that, with an integrated luminosity of $1 \mathrm{fb}^{-1}$ the LHC will generate $\approx 35000$ events per year, therefore the detection of any deviation from the SM prediction due to the SUSY EW and QCD one-loop corrections, at least in the early stages of the experiment, will be indeed very challenging. Also $A_{\mathrm{LR}}$ shows different behaviours in the two channels but, unfortunately, they are much greater in the s-channel, which, as we have just verified, has NLO corrections well beyond observability at $7 \mathrm{TeV}$. The same observability considerations can be applied to $A_{\mathrm{FB}}$ in the s-channel. 


\subsection{LHC at $14 \mathrm{TeV}$}

\subsubsection{The t-channel case}

The total cross section and asymmetries for this production mechanism are given in Tab. 5 .

\begin{tabular}{|c|c|c|}
\hline & $\sigma(\mathrm{pb})$ & L-R Asymmetry $(1-\delta)$ \\
\hline Born & 122.5 & $\delta=4.3491 \times 10^{-7}$ \\
\hline LS2 & $122.0(K=0.996)$ & $\delta=4.3668 \times 10^{-7}\left(1-K=1.77 \times 10^{-9}\right)$ \\
SPS1 & $122.1(K=0.997)$ & $\delta=4.3516 \times 10^{-7}\left(1-K=2.5 \times 10^{-10}\right)$ \\
\hline
\end{tabular}

Table 5: Numerical results for t-channel production at $14 \mathrm{TeV}$.

In this case the total cross section is significantly bigger than in the $7 \mathrm{TeV}$ case, and therefore we expect that it will be possible to extract much more information from experimental data. The $K$-factor for the integrated cross section is admittedly small, less than $1 \%$ for both benchmarks but, with the expected luminosity of $10 \mathrm{fb}^{-1}$, the production of single-top through this t-channel process will count $\sim 1 \mathrm{M}$ events and therefore it should be possible to detect a discrepancy in the expected number of SM events that could be interpreted as a hint of NP and thus boost the search of a SUSY resonance. As far as the $A_{\mathrm{LR}}$ is concerned, the top quark will emerge almost completely left-polarised, yet the detection of a one-loop induced discrepancy in the asymmetry will be again unlikely, the $K$-factor being close to one part in a billion or less.

The analysis of differential observables is now mandatory to better understand the origin of the corrections to the integrated cross section and Left-Right asymmetry. In Fig. 3 we have collected our results for the differential distributions of the cross section. It can be noticed that the corrections are always bigger in the LS2 scenario than in the SPS1a case and that in the transverse momentum distribution they can reach the $-10 \%$ limit for high values of the top $p_{t}$ (transverse momentum). However, the differential cross section is quite small in this limit, of the order of $0.01 \mathrm{pb} / \mathrm{TeV}$, and therefore observation of large one-loop corrections for events with high top transverse momentum is probably possible though quite difficult. It is also possible to see that the one-loop corrections in the other differential distributions are always quite small, below the $1 \%$ or so limit.

In Fig. 4 the one-loop corrections to the differential distributions for the Left-Right asymmetry are shown. Again, the corrections are generally bigger in the LS2 scenario than in the SPS1a case and can reach significant values for high top $p_{t}$, of $-8 \%$ or so. The $p_{t}$ distribution has another interesting feature: the asymmetry is bigger in the high top $p_{t}$ region, where, however, one-loop corrections are much less than $1 \%$.

\subsubsection{The s-channel case}

The total cross section and asymmetries for this production mechanism are given in Tab.6. 


\begin{tabular}{|c|c|c|c|}
\hline & $\sigma(\mathrm{pb})$ & L-R Asymmetry & F-B Asymmetry \\
\hline Born & 5.138 & 0.6861 & 0.53665 \\
\hline LS2 & $5.206(K=1.0132)$ & $0.6883(K=1.0032)$ & $0.53695(K=1.00056)$ \\
SPS1 & $5.145(K=1.0014)$ & $0.6869(K=1.0012)$ & $0.53676(K=1.00020)$ \\
\hline
\end{tabular}

Table 6: Numerical results for s-channel production at $14 \mathrm{TeV}$.

Single-top events from s-channel at the LHC will be significantly more than at the Tevatron, and this will allow a much more precise analysis of the process and of deviations to related observables due to new physics. The inclusive one-loop corrections we have obtained are at most of $\sim 1 \%$ in the LS2 scenario, but maybe enough to allow a small observable difference in the expected number of events. In constrast, the Left-Right asymmetry receives NLO corrections of the order of $0.1 \%$ in both the scenarios considered (here, unlike the general case, the effect of pure SUSY corrections is almost completely negligible). The same result holds for the one-loop corrections to the Forward-Backward asymmetry: the $K$-factor is very small and the effect practically undetectable with the s-channel cross section being of some pb.

The differential distributions for the cross section are shown in Fig. 5. In the s-channel case there are significant differences between the two scenarios considered: the corrections for LS2 are higher than those for SPS1a in the regions where the contributions to the total cross section are bigger, and this is the reason for the big difference in the $K$-factors for the integrated cross section in the two scenarios.

The same feature can be observed for the Left-Right asymmetry distributions, Fig. 6. where, however, the enhancement of the one-loop corrections from the LS2 scenario is milder and therefore the differences in the integrated asymmetry are smaller.

The corrections to differental quantities in the Forward-Backward asymmetry are qualitatively and quantitatively very similar to those for the Left-Right asymmetry, as it is possible to see in Fig. 7, thus the same comments as above apply in this case.

Considering all the differential distributions for the s-channel process, we can notice that they do not differ too much from each other between the two benchmarks and, as a general feature, they reach their maximum value in the invariant mass distribution for $M_{\mathrm{inv}} \gtrsim 700 \mathrm{GeV}$, in the transverse momentum distribution for $p_{T} \gtrsim 300 \mathrm{GeV}$ and in the top rapidity for $y_{t} \gtrsim 1.5$.

To better understand the origin of the corrections and why there are peaks for certain values of $M_{\mathrm{inv}}, p_{T}$ and $y_{t}$ we have computed (here, limitedly to the s-channel) the SUSY EW and QCD corrections for the cross section separately and plotted the related differential quantities in Figs. 8 and 9 for the two benchmark scenarios. It is possible to see that the corrections are dominated by the SUSY QCD contribution, while the EW counterpart provides small contributions to the total corrections. In both bench- 
mark scenarios, however, the EW contribution shows peculiar features in the invariant mass distribution, given by peaks and troughs in the correction, and a closer inspection of these threshold effects reveals that they are situated in correspondence of $M_{\chi_{i}^{ \pm}}^{2}$. Therefore charginos could play an interesting role in the determination of SUSY effects in s-channel single top production processes. Going back to the dominant QCD contribution, the only parameter which enter the QCD correction alone is the gluino mass $\left(m_{\tilde{g}}=607 \mathrm{GeV}\right.$ in SPS1a and $m_{\tilde{g}}=392 \mathrm{GeV}$ in LS2), thus we can argue that the one-loop corrections are sensitive mostly to this parameter. Smaller gluino masses shift the SUSY QCD peak in the corrections towards regions where the differential distributions have higher values and therefore the integrated quantities will be affected more by NLO corrections. This means that observing higher pure SUSY one-loop corrections would point towards scenarios with lighter gluino and viceversa.

To conclude this section, we are aware that recent measurements have slightly changed the value of the top mass[14]. Nevertheless, we have verified that any change (within a reasonable range) in this value has practically no effect on one-loop corrections, as it is possible to see in Fig. 10,

\section{Conclusions}

In summary, QCD and EW corrections through one-loop level originating from SUSY and affecting the cross section for ' $t+$ jet' final states at the LHC are always small at inclusive level, at both energies of 7 and $14 \mathrm{TeV}$. However, they appear sizable (up to ten percent) and detectable (after $\mathcal{O}(100) \mathrm{fb}^{-1}$ of luminosity) at differential level, if the collider energy is $14 \mathrm{TeV}$, while for $7 \mathrm{TeV}$ they will presumably not be accessible. Such large corrections also appear in the Left-Right and Forward-Backward (when defineable) asymmetries, again, at the differential but not inclusive level. The bulk of the higher order effects comes from QCD, with a much smaller, yet very sensitive to SUSY parameters, EW component. The corrections discussed here are typically larger for the s-channel mode than for the t-channel one, which renders them more difficult to access as the former is subleading with respect to the latter at the LHC. Altogether though, our results point to the relevance of SUSY one-loop effects onto ' $t+$ jet' final states for the LHC running at design energy and luminosity. In presence of high statistic samples, some typical SUSY parameter dependencies could possibly be extracted, in either mSUGRA or the MSSM.

\section{Acknowledgements}

Both GM and LP acknowledge financial support from the Royal Society (London, UK) in the form of a travel grant to visit Southampton. SM is financially supported in part 
by the scheme 'Visiting Professor - Azione D - Atto Integrativo tra la Regione Piemonte e gli Atenei Piemontesi'. GM and LP would like to thank Matteo Beccaria, Claudio Verzegnassi, and Fernand Renard for valuable suggestions and discussions during earlier work on single top production.

\section{References}

[1] W. Beenakker, W. L. van Neerven, R. Meng, G. A. Schuler and J. Smith, Nucl. Phys. B 351 (1991) 507;

W. Bernreuther, A. Brandenburg, Z. G. Si and P. Uwer, Int. J. Mod. Phys. A 18 (2003) 1357;

N. Kidonakis and R. Vogt, Phys. Rev. D 78 (2008) 074005;

M. Cacciari, S. Frixione, M. L. Mangano, P. Nason and G. Ridolfi, JHEP 0809 (2008) 127;

M. Czakon and A. Mitov, Nucl. Phys. B 824 (2010) 111;

K. Melnikov and M. Schulze, JHEP 0908 (2009) 049;

A. Bredenstein, A. Denner, S. Dittmaier and S. Pozzorini, JHEP 1003 (2010) 021.

[2] S. Moretti, M. R. Nolten and D. A. Ross, Phys. Lett. B 639 (2006) 513 [Erratum-ibid. B 660 (2008) 607];

J. H. Kuhn, A. Scharf and P. Uwer, Eur. Phys. J. C 51 (2007) 37;

W. Hollik and M. Kollar, Phys. Rev. D 77 (2008) 014008;

W. Bernreuther, M. Fucker and Z. G. Si, Nuovo Cim. 123B (2008) 1036.

[3] B. W. Harris, E. Laenen, L. Phaf, Z. Sullivan and S. Weinzierl, Phys. Rev. D 66 (2002) 054024;

S. Frixione, E. Laenen, P. Motylinski and B. R. Webber, JHEP 0603 (2006) 092;

N. Kidonakis, Phys. Rev. D 75 (2007) 071501;

S. Frixione, E. Laenen, P. Motylinski, B. R. Webber and C. D. White, JHEP 0807 (2008) 029;

J. M. Campbell, R. Frederix, F. Maltoni and F. Tramontano, Phys. Rev. Lett. 102 (2009) 182003;

S. Heim, Q. H. Cao, R. Schwienhorst and C. P. Yuan, Phys. Rev. D 81 (2010) 034005.

[4] M. Beccaria, C. M. Carloni Calame, G. Macorini, E. Mirabella, F. Piccinini, F. M. Renard and C. Verzegnassi, Phys. Rev. D 77 (2008) 113018; 
M. Beccaria, G. Macorini, F. M. Renard and C. Verzegnassi, Phys. Rev. D 74 (2006) 013008;

M. Beccaria, C. M. Carloni Calame, G. Macorini, G. Montagna, F. Piccinini, F. M. Renard and C. Verzegnassi, Eur. Phys. J. C 53 (2008) 257.

[5] D. A. Ross and M. Wiebusch, JHEP 0711 (2007) 041.

[6] W. Bernreuther, M. Fuecker and Z. G. Si, Phys. Rev. D 74 (2006) 113005.

[7] M. Beccaria, F. M. Renard and C. Verzegnassi, Phys. Rev. D 71 (2005) 033005;

C. S. Li, R. J. Oakes and J. M. Yang, Phys. Rev. D 55 (1997) 5780.

[8] J. Pumplin, D. R. Stump, J. Huston, H. L. Lai, P. M. Nadolsky and W. K. Tung, JHEP 0207012 (2002) 012.

[9] A. Djouadi, J. L. Kneur and G. Moultaka, Comput. Phys. Commun. 176 (2007) 426.

[10] V. D. Barger, N. G. Deshpande, J. L. Rosner and K. Whisnant, Phys. Rev. D 35 (1987) 2893.

[11] O. Brein, Comput. Phys. Commun. 170 (2005) 42.

[12] B. C. Allanach et al., Eur. Phys. J. C 25 (2002) 113.

[13] M. Beccaria, G. Macorini, F. M. Renard and C. Verzegnassi, Phys. Rev. D 74 (2006) 013008 .

[14] K. Nakamura et al. (Particle Data Group), Journal of Physics G 37, 075021 (2010) 

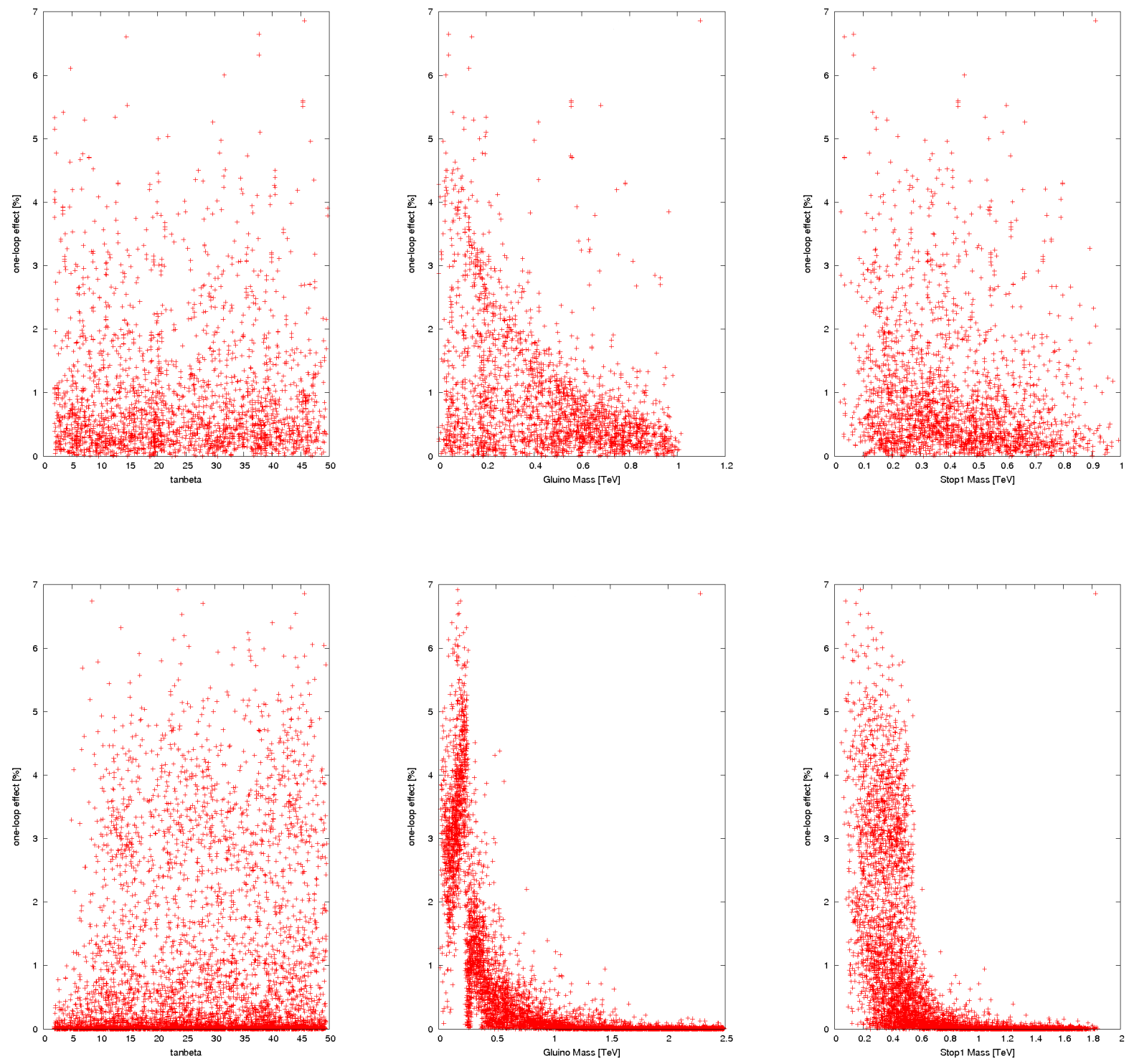

Figure 1: Scan over three relevant SUSY parameters $\left(\tan \beta, m_{\tilde{g}}\right.$ and $\left.m_{\tilde{t}}\right)$ in the context of the MSSM (upper panels) and mSUGRA (lower panels) to search for maxima of one-loop effects. 

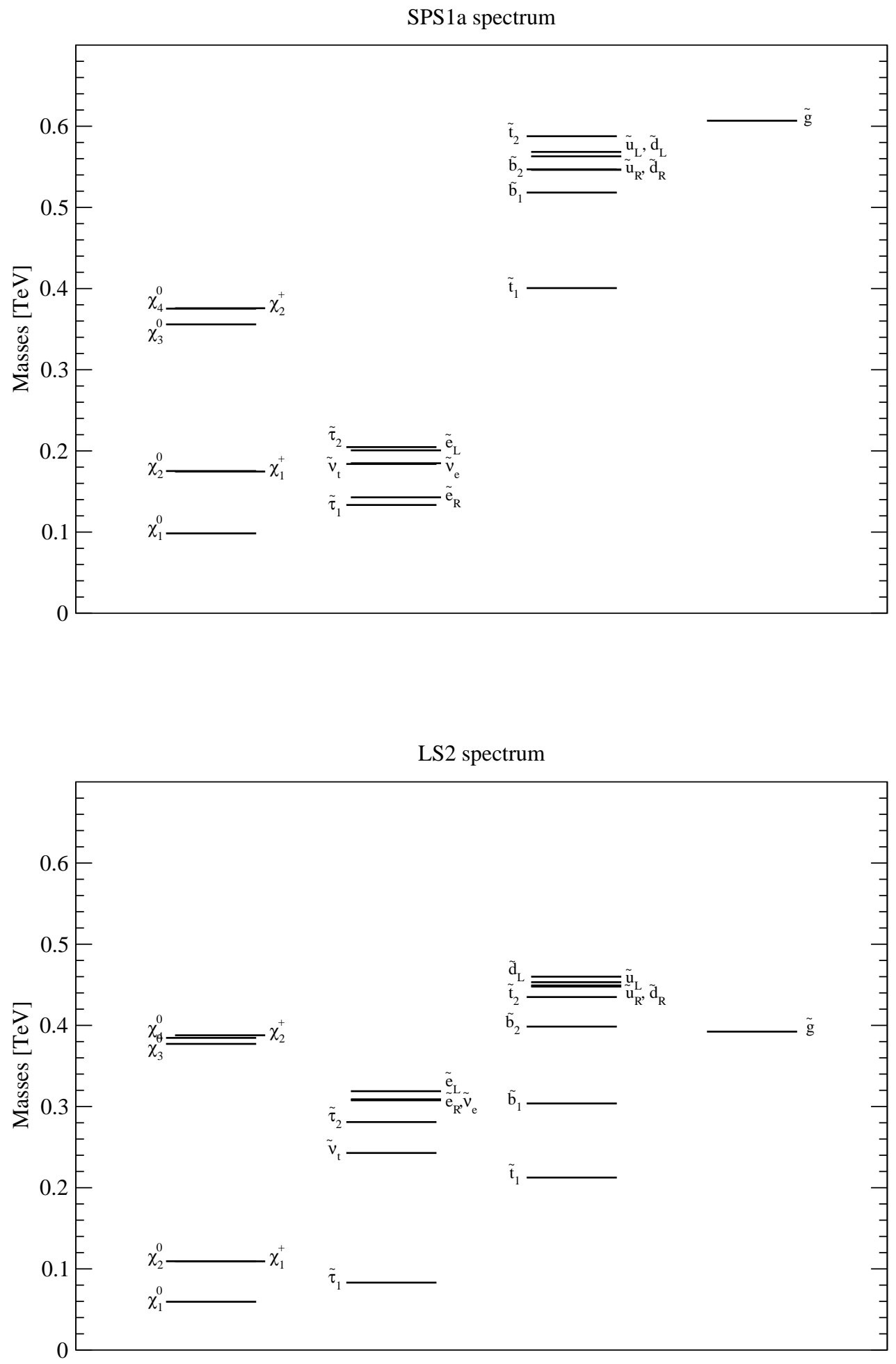

Figure 2: The SPS1a and LS2 mass spectra. 

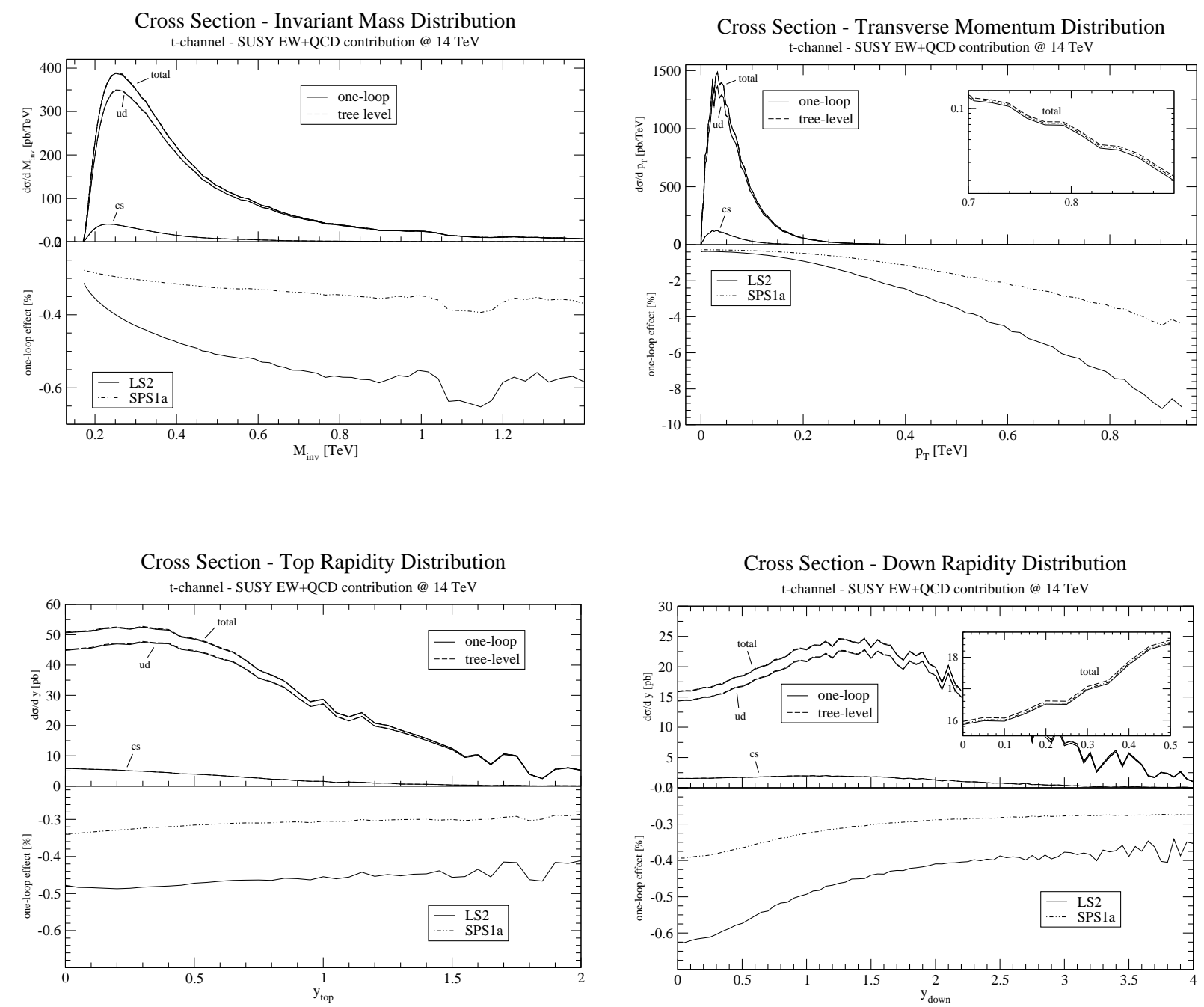

Figure 3: Differential distributions of the cross section in $t$-channel at $14 \mathrm{TeV}$. In this and in the following Figs., the small panels focus on the range where the one-loop effects are higher, and they are shown only when the corrections from LS2 and SPS1a are distinguishable. 

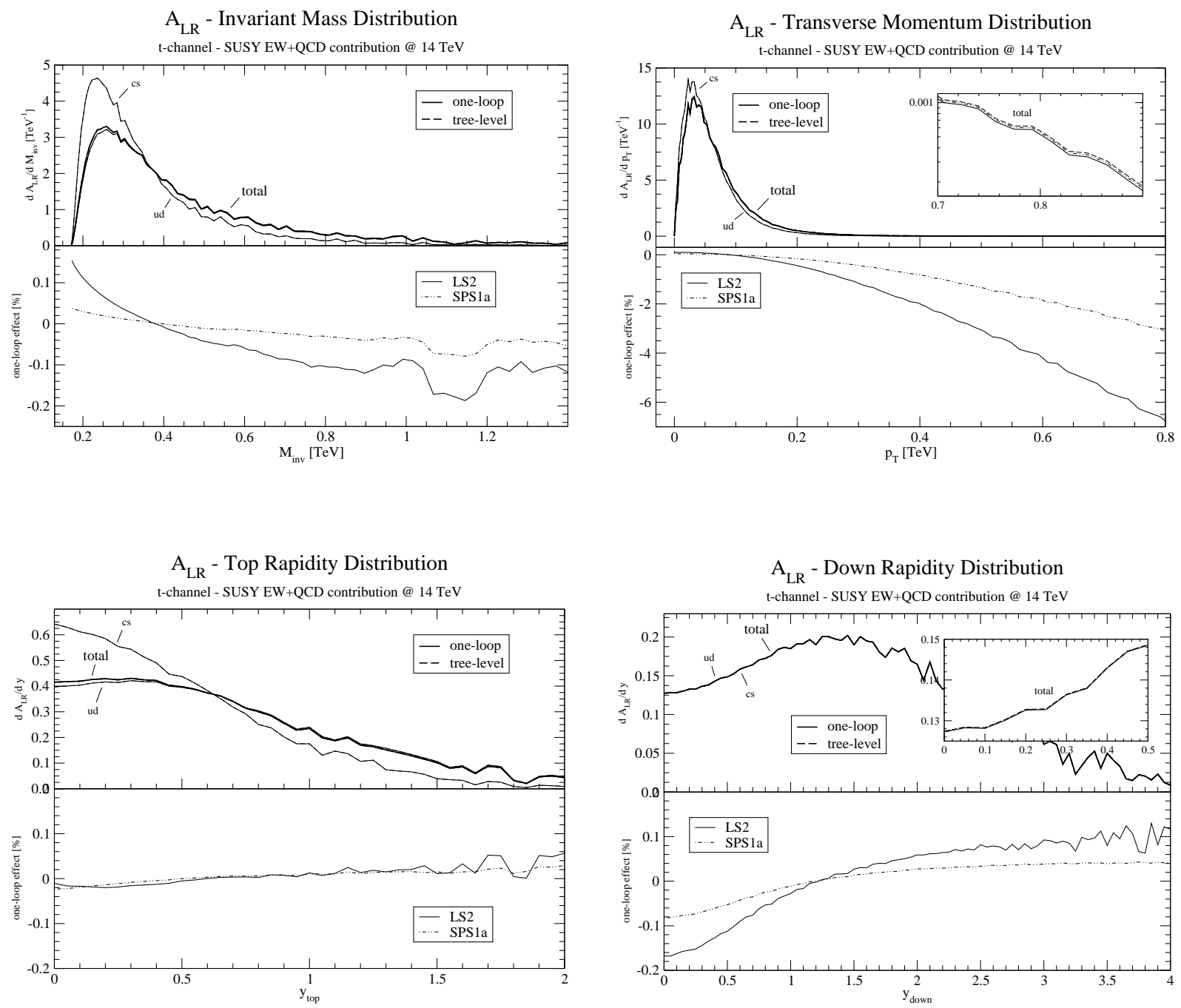

Figure 4: Differential distributions of the Left-Right asymmetry in $t$-channel at $14 \mathrm{TeV}$. 

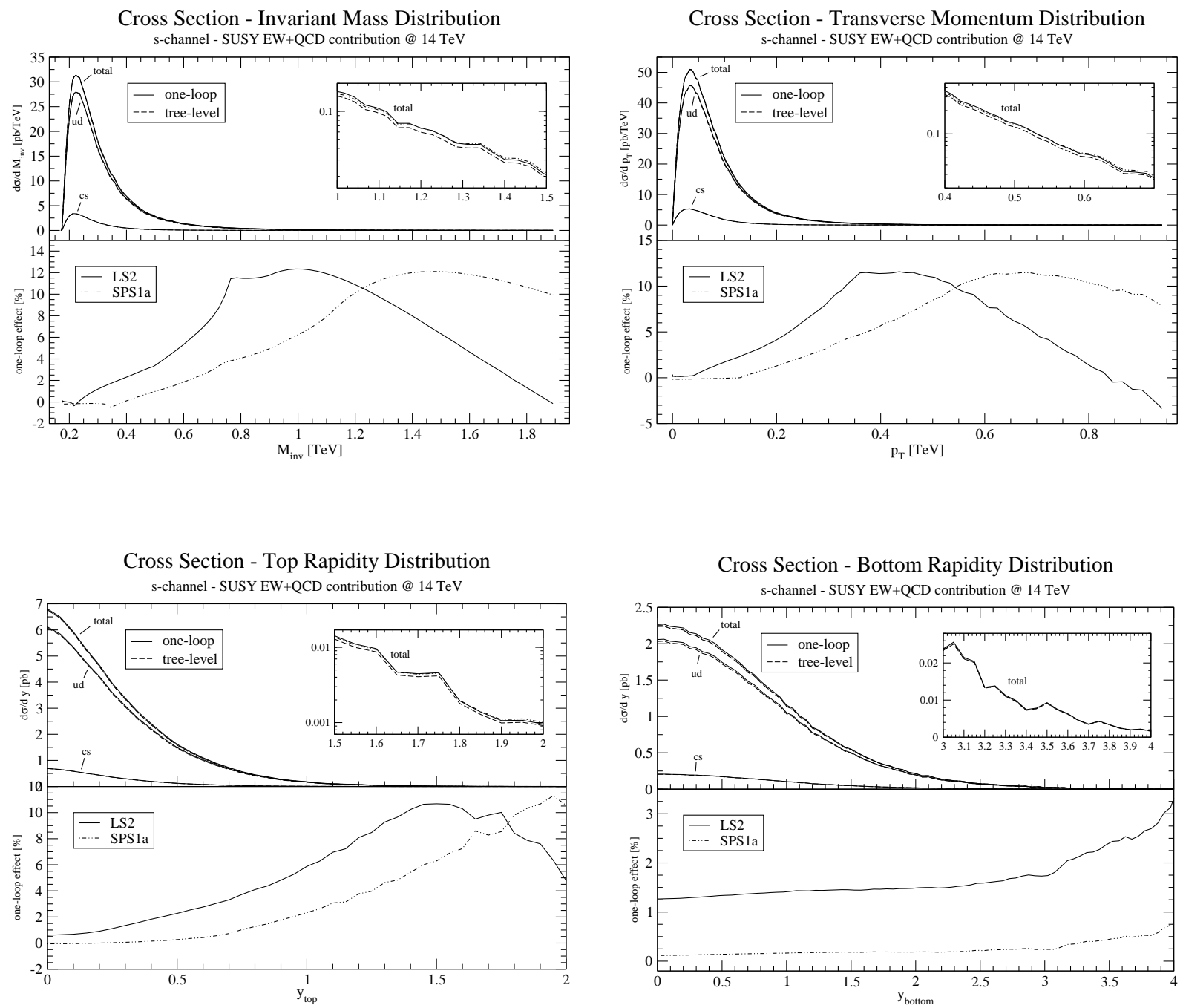

Figure 5: Differential distributions of the cross section in $s$-channel at $14 \mathrm{TeV}$. 

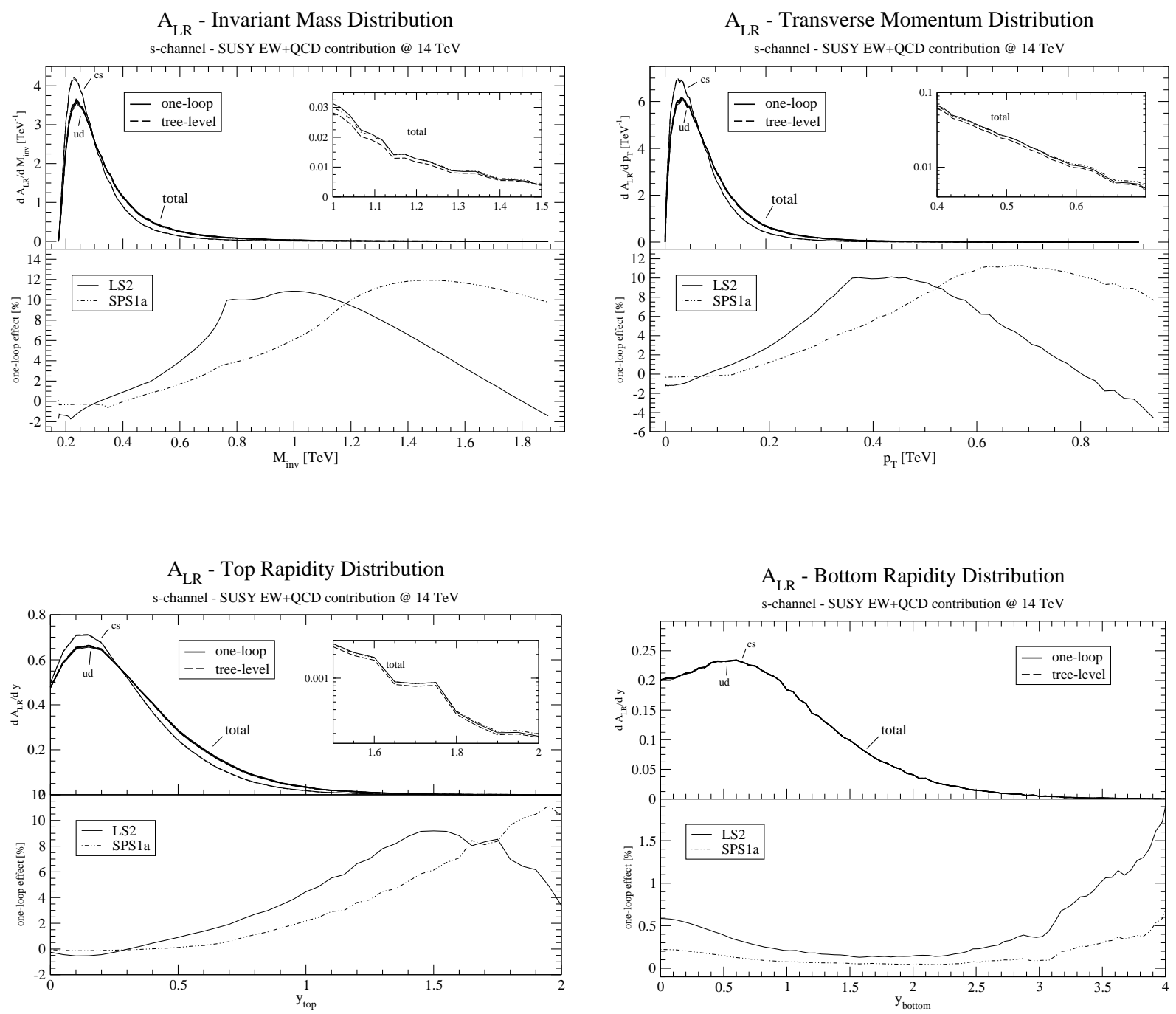

Figure 6: Differential distributions of the Left-Right asymmetry in s-channel at $14 \mathrm{TeV}$. 

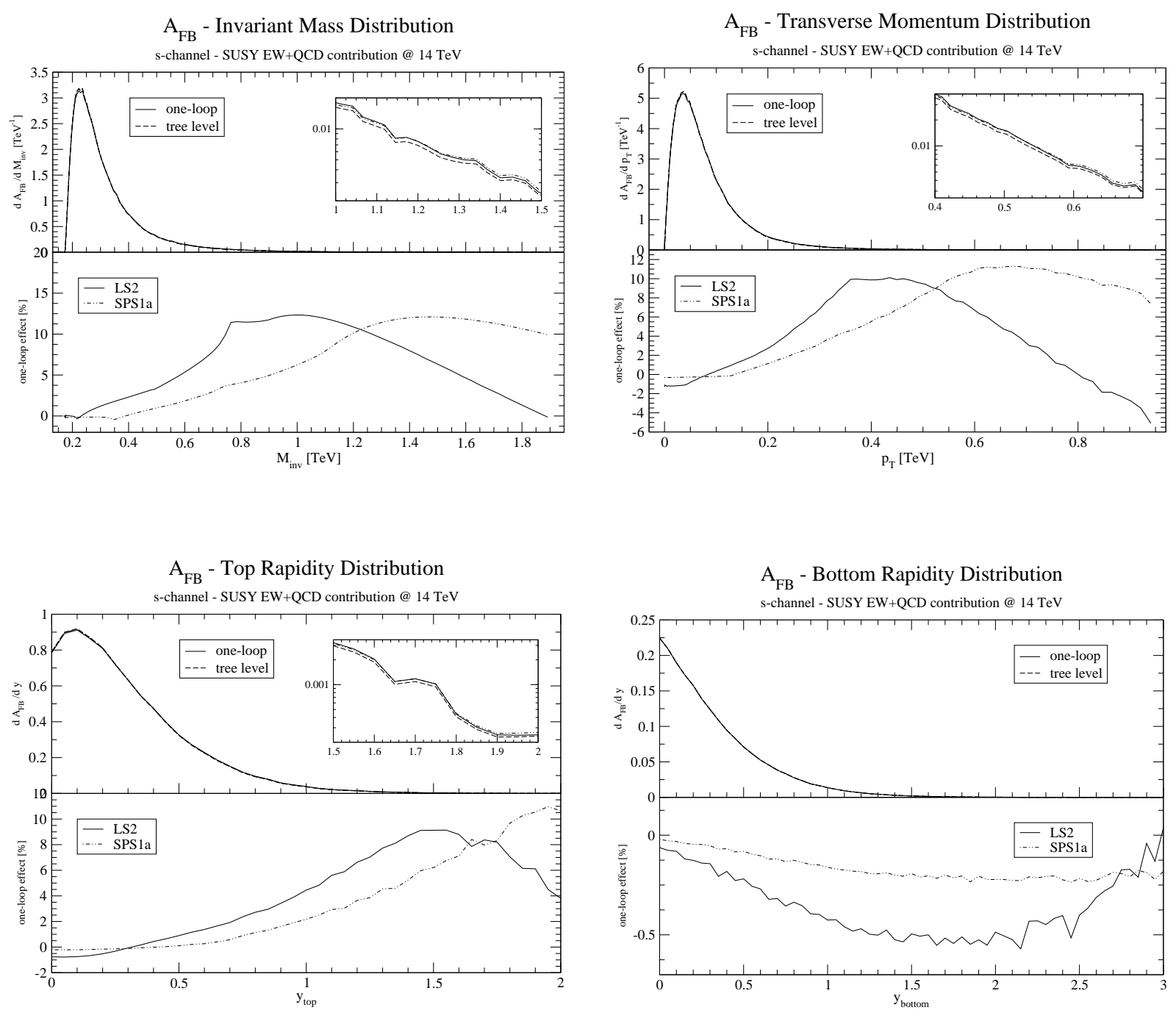

Figure 7: Differential distributions of the Forward-Backward asymmetry in $s$-channel at $14 \mathrm{TeV}$. 

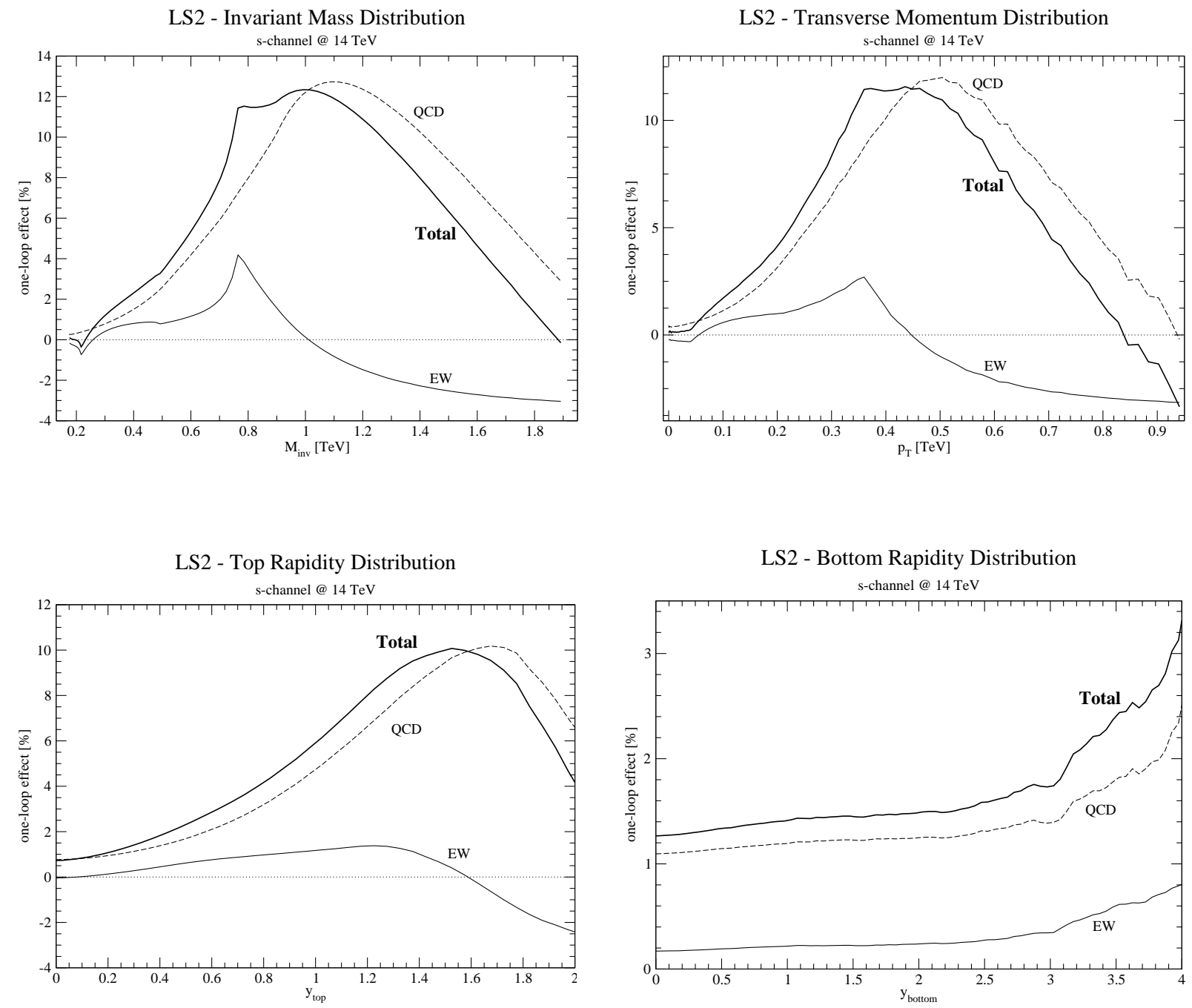

Figure 8: SUSY EW and QCD corrections to differential distributions of the cross section in $s$-channel at $14 \mathrm{TeV}$ for LS2. 

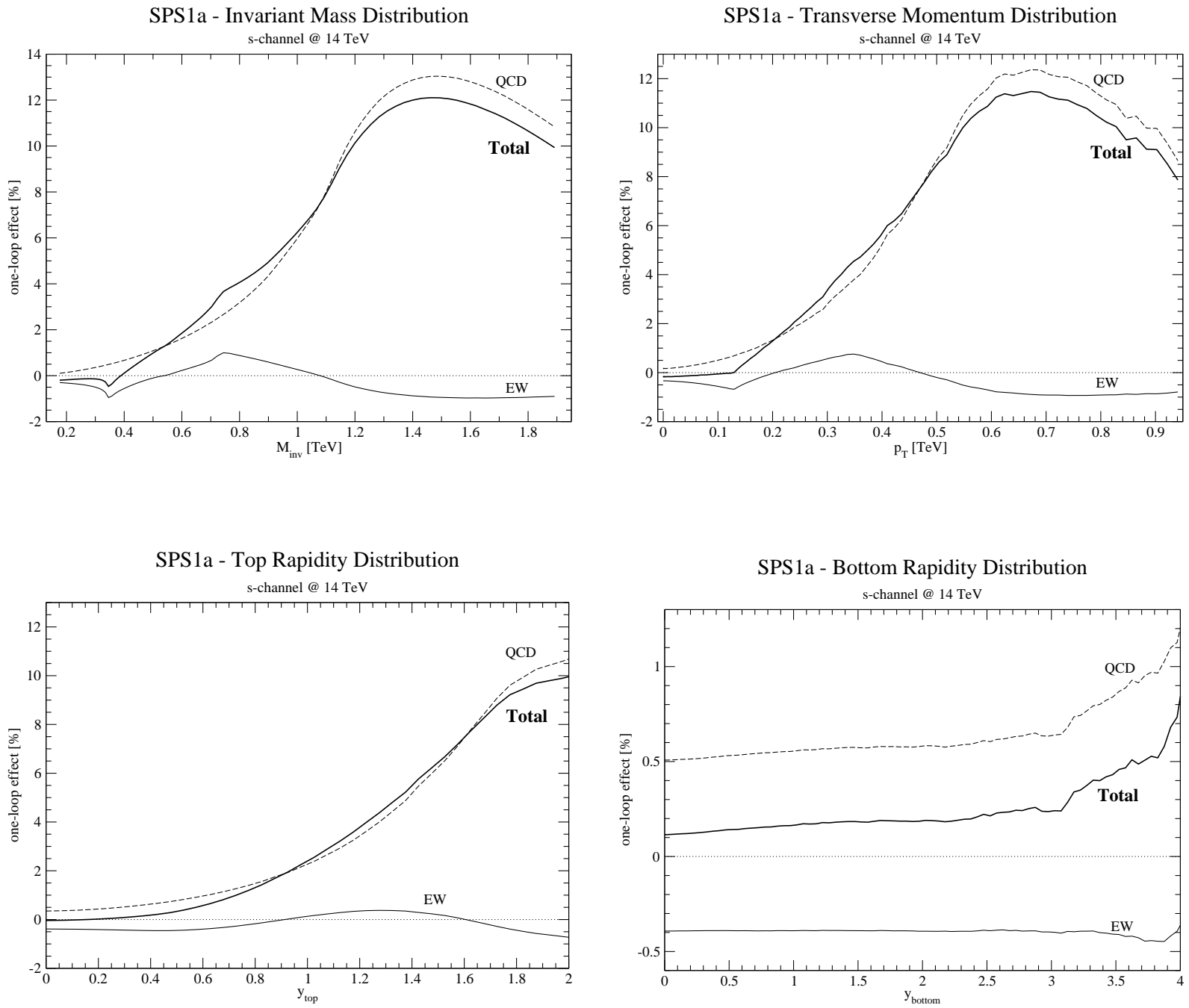

Figure 9: SUSY EW and QCD corrections to differential distributions of the cross section in $s$-channel at $14 \mathrm{TeV}$ for SPS1a. 


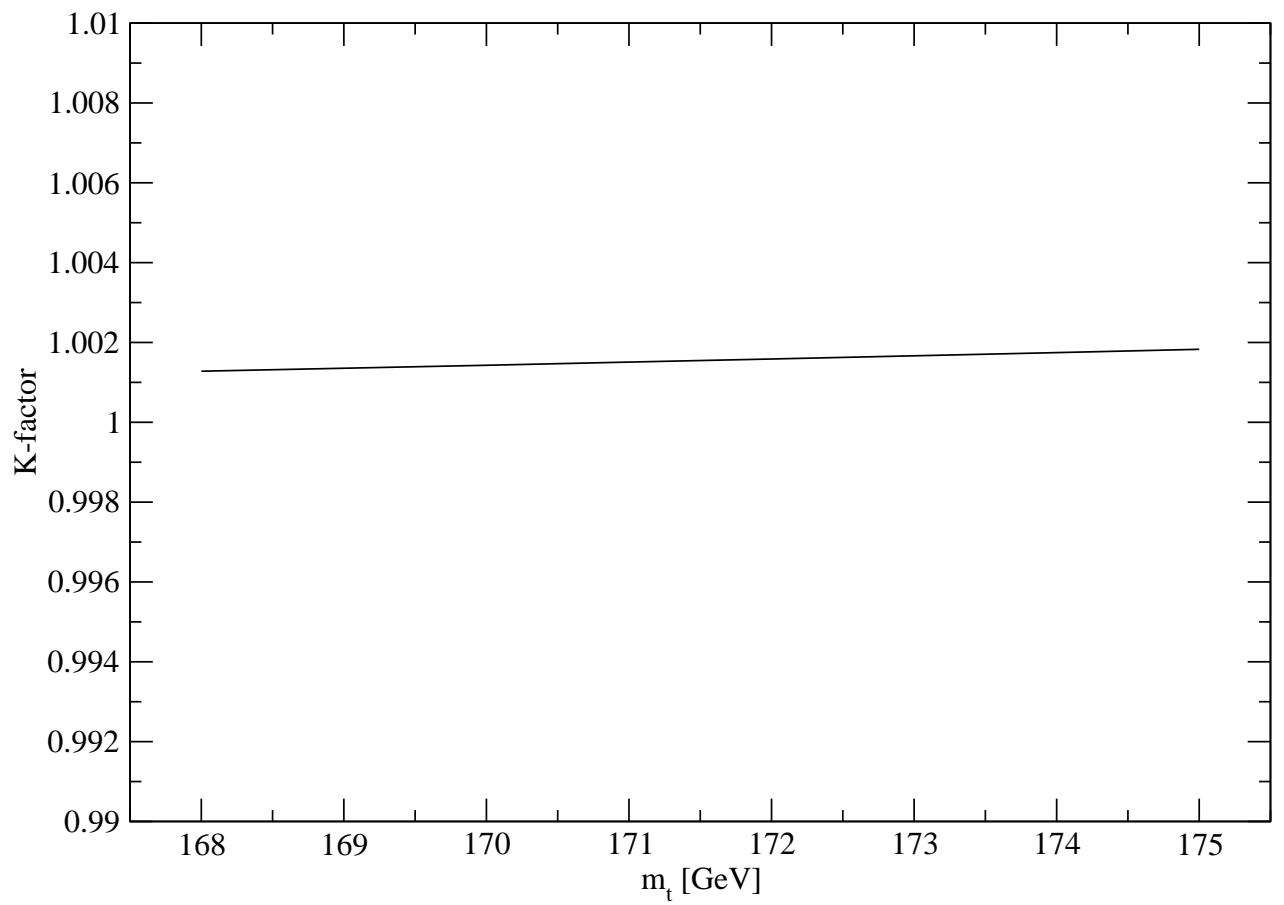

Figure 10: K-factor dependence on the top mass in s-channel at $14 \mathrm{TeV}$ for SPS1a. 